\section{Alimentação escolar e agricultura familiar: análise de recursos empregados na compra de alimentos}

\author{
School meals and family farming: analysis of \\ funds spent in food purchases
}

\section{Alimentación escolar y agricultura familiar: análisis de recursos empleados en la compra de alimentos}

Lana Raysa da Silva Araujo 1

Andrea Nunes Mendes de Brito 1

Malvina Thais Pacheco Rodrigues 1

Márcio Dênis Medeiros Mascarenhas 1

Regilda Saraiva dos Reis Moreira-Araujo 1

doi: 10.1590/0102-311X00004819

\section{Resumo}

O objetivo do estudo foi verificar a adequação da utilização de recursos financeiros para a compra de alimentos provenientes da agricultura familiar no âmbito do Programa Nacional de Alimentação Escolar (PNAE) nas capitais de estados brasileiros e no Distrito Federal. Estudo descritivo, com análise de dados secundários obtidos na página eletrônica do Fundo Nacional de Desenvolvimento para a Educação (FNDE) no espaço dedicado às informações sobre agricultura familiar. Foram investigados os valores repassados pelo FNDE e percentual utilizado na compra de alimentos da agricultura familiar para aquisição de alimentação escolar em capitais dos estados brasileiros e no Distrito Federal no período de 2011 a 2017. Os dados foram coletados em maio de 2019. Das 27 cidades avaliadas, cerca de um terço (33,3\%) apresentou compra acima do mínimo recomendado (30\%). A Região Norte cumpriu o recomendado $(39,4 \%)$ enquanto a Sudeste apresentou a menor porcentagem de compra (6,4\%). De 2011 a 2017, percebeu-se aumento nos valores totais investidos e no número de cidades compradoras de alimentos da agricultura familiar. Dentre as capitais avaliadas, Boa Vista (Roraima) foi a que mais utilizou recursos para a aquisição de alimentos da agricultura familiar (56,6\%). Em 2017, as prefeituras de Boa Vista e Aracaju (Sergipe) ofereceram contrapartida financeira adicionada aos recursos fornecidos pelo FNDE para a compra desses alimentos. Concluiu-se que, no período de 2011 a 2017, apenas um terço das capitais avaliadas utilizou adequadamente a porcentagem de recursos financeiros para a compra de alimentos da agricultura familiar, embora tenha sido percebido aumento gradual na compra desses alimentos, especialmente nas capitais do Norte e Nordeste do Brasil.

Agricultura; Alimentação Escolar; Financiamento Governamental

\author{
Correspondência \\ L. R. S. Araujo \\ Programa de Pós-graduação em Saúde e Comunidade, \\ Universidade Federal do Piauí. \\ Av. Frei Serafim 2280, Teresina, PI 64210-360, Brasil. \\ lannaraysa@hotmail.com \\ 1 Programa de Pós-graduação em Saúde e Comunidade, \\ Universidade Federal do Piauí, Teresina, Brasil.
}




\section{Introdução}

A agricultura familiar é a principal forma de produção agrícola de diversas cidades brasileiras e constitui uma estratégia de desenvolvimento econômico, social, cultural e sustentável. Com o incentivo adequado, esta produção contribui com a redução do desemprego, fome e desnutrição, além de promover o consumo de alimentos regionais e saudáveis pela população 1,2, por meio da oferta de frutas, hortaliças, fibras e cereais integrais 3 .

No Brasil, a aquisição de alimentos da agricultura familiar tem sido incentivada por meio de políticas promotoras de alimentação e nutrição. O Programa Nacional de Alimentação Escolar (PNAE) visa favorecer a alimentação adequada por intermédio de estratégias promotoras de crescimento e desenvolvimento, bem como da alimentação saudável 4,5. Por intermédio do PNAE, o Fundo Nacional de Desenvolvimento para a Educação (FNDE) repassa recursos financeiros aos estados, Distrito Federal e municípios para a compra de gêneros alimentícios designados ao atendimento das necessidades dos estudantes da Rede Pública de Ensino 6, com a obrigatoriedade de utilização de, no mínimo, 30\% desses recursos para a compra de alimentos da agricultura familiar conforme diretrizes estabelecidas na Lei no 11.947/2009 7,8.

Este estudo teve como objetivo verificar a adequação da utilização de recursos financeiros para a compra de alimentos provenientes da agricultura familiar nas capitais dos estados brasileiros e no Distrito Federal.

\section{Métodos}

Estudo descritivo, com análise de dados secundários obtidos na página de Internet do FNDE, no espaço dedicado às informações sobre agricultura familiar (https://www.fnde.gov.br/index.php/ programas/pnae/pnae-consultas/pnae-dados-da-agricultura-familiar).

Foram investigados o total de recursos repassados pelo FNDE e o percentual utilizado na compra de alimentos da agricultura familiar para aquisição de alimentação escolar em capitais dos estados brasileiros e no Distrito Federal, no período de 2011 a 2017. A coleta de dados ocorreu em maio de 2019.

Calculou-se a porcentagem de compra de gêneros alimentícios procedentes da agricultura familiar, tendo como referência o mínimo de 30\% preconizado pela Lei no 11.947/2009, o valor gasto por região durante cada ano avaliado e o percentual de adequação de todas as cidades por ano. A porcentagem de adequação de compra de alimentos da agricultura familiar (mínimo de 30\%) foi descrito segundo capitais e regiões geográficas. Os dados foram analisados no software IBM SPSS 20.0 (https://www.ibm.com/).

Por se tratar de dados de domínio público, foi dispensada a submissão ao Comitê de Ética em Pesquisa.

\section{Resultados}

De 2011 a 2017, o FNDE transferiu R\$2.746.866.252,41 para a compra de alimentação escolar nas 26 capitas de estados e no Distrito Federal. Deste total, R\$ 357.443.960,12 (13\%) foram utilizados na compra de alimentos da agricultura familiar.

Das 27 cidades avaliadas, cerca de um terço $(33,3 \%)$ utilizou o mínimo de $30 \%$ dos recursos financeiros para a compra de produtos da agricultura familiar. Somente a Região Norte cumpriu a recomendação da legislação vigente (39,4\%). A Sudeste apresentou a menor porcentagem de compra $(6,4 \%)$. Boa Vista (Roraima) foi a capital que mais adquiriu alimentos da agricultura familiar $(56,6 \%)$ enquanto o Rio de Janeiro efetuou a menor utilização de recursos $(0,1 \%)$ (Tabela 1$)$.

Houve aumento nos valores totais investidos no período avaliado, com exceção do ano 2016, quando houve decréscimo. Ao comparar o valor total investido em 2011 e 2017, foi evidenciado aumento em todas as macrorregiões, com destaque para as regiões Norte e Nordeste que apresen- 


\section{Tabela 1}

Valores empregados na aquisição de gêneros alimentícios da agricultura familiar para o Programa Nacional de Alimentação Escolar (PNAE), segundo macrorregiões geográficas, capitais de estado e Distrito Federal. Brasil, 2011-2017.

\begin{tabular}{|c|c|c|c|}
\hline & \multirow[t]{2}{*}{ Valor total repassado $(\mathbf{R} \$)$} & \multicolumn{2}{|c|}{$\begin{array}{l}\text { Valor empregado na aquisição de alimentos } \\
\text { da agricultura familiar }\end{array}$} \\
\hline & & $\mathbf{R} \$$ & $\%$ \\
\hline Total & $2.746 .866 .252,41$ & $357.443 .960,12$ & 13,0 \\
\hline Norte & $298.778 .225,49$ & $117.728 .735,05$ & 39,4 \\
\hline Rio Branco (Acre) & $15.131 .804,65$ & $5.519 .541,87$ & 36,5 \\
\hline Macapá (Amapá) & $18.405 .567,98$ & $5.646 .178,86$ & 30,7 \\
\hline Manaus (Amazonas) & $138.977 .135,59$ & $58.152 .472,10$ & 41,8 \\
\hline Belém (Pará) & $43.480 .164,23$ & $15.883 .692,07$ & 36,5 \\
\hline Porto Velho (Rondônia) & $26.909 .824,92$ & $5.114 .455,22$ & 19,0 \\
\hline Boa Vista (Roraima) & 17.869.036,95 & 10.116.675,06 & 56,6 \\
\hline Palmas (Tocantins) & $38.004 .691,17$ & $17.295 .719,87$ & 45,5 \\
\hline Nordeste & $569.457 .031,87$ & $72.651 .378,05$ & 12,8 \\
\hline São Luís (Maranhão) & $94.658 .357,95$ & $14.874 .028,87$ & 15,7 \\
\hline Teresina (Piauí) & $62.183 .385,36$ & $23.931 .203,62$ & 38,5 \\
\hline Fortaleza (Ceará) & $136.589 .953,05$ & $7.035 .575,83$ & 5,2 \\
\hline Natal (Rio Grande do Norte) & $29.577 .678,51$ & $1.181 .399,58$ & 4,0 \\
\hline João Pessoa (Paraíba) & $39.780 .987,83$ & $4.112 .511,52$ & 10,3 \\
\hline Recife (Pernambuco) & $68.555 .541,34$ & $1.208 .853,10$ & 1,8 \\
\hline Maceió (Alagoas) & $26.500 .207,42$ & $685.494,44$ & 2,6 \\
\hline Aracaju (Sergipe) & $13.632 .006,14$ & $2.720 .701,65$ & 20,0 \\
\hline Salvador (Bahia) & $97.978 .914,27$ & $16.901 .609,44$ & 17,3 \\
\hline Sudeste & $1.246 .263 .207,65$ & $80.085 .038,64$ & 6,4 \\
\hline Vitória (Espírito Santo) & $35.217 .239,34$ & $11.684 .442,25$ & 33,2 \\
\hline Belo Horizonte (Minas Gerais) & $143.861 .386,14$ & $15.154 .131,76$ & 10,5 \\
\hline Rio de Janeiro (Rio de Janeiro) & $449.405 .104,89$ & $495.469,42$ & 0,1 \\
\hline São Paulo (São Paulo) & $617.779 .477,28$ & $52.750 .995,21$ & 8,5 \\
\hline Sul & $196.731 .481,48$ & $22.073 .624,17$ & 11,2 \\
\hline Curitiba (Paraná) & $115.207 .432,16$ & $3.031 .614,39$ & 2,6 \\
\hline Porto Alegre (Rio Grande do Sul) & $55.945 .866,88$ & $13.828 .149,97$ & 24,7 \\
\hline Florianópolis (Santa Catarina) & $25.578 .182,44$ & $5.213 .859,81$ & 20,4 \\
\hline Centro-oeste & $435.636 .305,92$ & $64.905 .184,21$ & 14,9 \\
\hline Distrito Federal & $253.633 .755,67$ & $12.748 .225,98$ & 5,0 \\
\hline Goiânia (Goiás) & $75.014 .902,36$ & $32.087 .983,86$ & 42,8 \\
\hline Cuiabá (Mato Grosso) & $42.750 .980,19$ & $9.567 .018,13$ & 22,4 \\
\hline Campo Grande (Mato Grosso do Sul) & $64.236 .667,70$ & $10.501 .956,24$ & 16,4 \\
\hline
\end{tabular}

Fonte: Fundo Nacional de Desenvolvimento para Educação (FNDE), 2019.

taram evolução positiva e consistente do valor gasto por ano na compra de alimentos da agricultura familiar, no período estudado (Tabela 2).

Houve ampliação do número de cidades compradoras de alimentos da agricultura familiar, variando de 14 em 2011 para 27 em 2017. O ano de 2015 apresentou a maior porcentagem de compra de agricultura familiar nos anos avaliados. Manaus (Amazonas) foi a única capital que teve crescimento constante da aquisição desses alimentos em todo o período estudado. No ano de 2016, as cidades de Boa Vista e Maceió (Alagoas) utilizaram todo o recurso fornecido pelo FNDE com produtos da agricultura familiar. Em 2017, as prefeituras de Boa Vista e Aracaju (Sergipe) ofereceram contrapartida financeira para a compra de alimentos oriundos da agricultura familiar (Tabela 3). 
Tabela 2

Valores (R\$) empregados na aquisição de gêneros alimentícios da agricultura familiar para o Programa Nacional de Alimentação Escolar (PNAE), segundo macrorregiões geográficas, capitais de estado e Distrito Federal. Brasil, 2011-2017.

\begin{tabular}{|c|c|c|c|c|c|c|c|}
\hline & 2011 & 2012 & 2013 & 2014 & 2015 & 2016 & 2017 \\
\hline Total & $10.670 .960,68$ & $28.181 .951,26$ & $42.117 .732,88$ & $57.867 .195,73$ & $78.706 .562,76$ & $63.919 .307,53$ & $75.980 .249,28$ \\
\hline Norte & $4.024 .076,89$ & $9.770 .568,51$ & $16.177 .538,49$ & $16.436 .710,36$ & $22.082 .222,02$ & $24.050 .355,87$ & $25.187 .262,91$ \\
\hline Rio Branco (Acre) & $517.869,68$ & $445.427,14$ & $736.400,70$ & $464.057,22$ & $1.047 .920,67$ & $1.014 .937,49$ & $1.292 .928,97$ \\
\hline Macapá (Amapá) & - & $693.639,93$ & $703.093,76$ & $12.000,00$ & $1.682 .967,90$ & $978.233,54$ & $1.576 .243,73$ \\
\hline Manaus (Amazonas) & $1.019 .322,51$ & $5.318 .759,18$ & $8.039 .770,22$ & $8.212 .650,64$ & $10.434 .968,59$ & $11.895 .123,76$ & $13.231 .877,20$ \\
\hline Belém (Pará) & $1.661 .935,95$ & $901.560,00$ & $2.908 .164,93$ & 3.102.643,39 & $2.041 .119,80$ & $2.590 .114,04$ & $2.678 .153,96$ \\
\hline Porto Velho (Rondônia) & - & - & $788.833,57$ & $246.141,66$ & $1.159 .731,48$ & $1.885 .515,85$ & $1.034 .232,66$ \\
\hline Boa Vista (Roraima) & - & $592.503,00$ & $590.037,49$ & $1.685 .715,56$ & $2.672 .577,32$ & $2.392 .953,75$ & $2.182 .887,94$ \\
\hline Palmas (Tocantins) & $824.948,75$ & $1.818 .679,26$ & $2.411 .237,82$ & $2.713 .501,89$ & $3.042 .936,26$ & $3.293 .477,44$ & $3.190 .938,45$ \\
\hline Nordeste & $1.650 .608,78$ & $7.559 .157,89$ & $8.827 .469,23$ & $8.809 .243,04$ & $12.040 .226,94$ & $14.398 .696,65$ & $19.365 .975,52$ \\
\hline São Luís (Maranhão) & - & - & $4.301 .986,40$ & - & $2.333 .287,22$ & $5.392 .744,19$ & $2.846 .011,06$ \\
\hline Teresina (Piauí) & $1.330 .104,78$ & $2.988 .986,27$ & $2.814 .632,08$ & $4.156 .311,55$ & $3.501 .884,2$ & $4.365 .131,70$ & $4.774 .153,04$ \\
\hline Fortaleza (Ceará) & - & $763.916,47$ & $8.425,86$ & $927.557,25$ & $407.407,83$ & $2.198 .513,18$ & $2.729 .755,24$ \\
\hline Natal (Rio Grande do & - & - & $90.192,20$ & $227.995,86$ & $423.893,11$ & $243.919,01$ & $195.399,40$ \\
\hline \multicolumn{8}{|l|}{ Norte) } \\
\hline João Pessoa (Paraíba) & $54.124,00$ & - & $795.438,53$ & - & $115.691,21$ & $914.017,52$ & $2.233 .240,26$ \\
\hline Recife (Pernambuco) & - & - & - & - & - & - & $1.208 .853,10$ \\
\hline Maceió (Alagoas) & - & - & $4.800,00$ & - & $300.629,46$ & $170.977,02$ & $209.087,96$ \\
\hline Aracaju (Sergipe) & - & $295.046,66$ & $303.249,56$ & $166.534,38$ & $156.933,91$ & $837.486,25$ & $961.450,89$ \\
\hline Salvador (Bahia) & $266.380,00$ & $3.511 .208,49$ & $508.744,60$ & $3.330 .844,00$ & $4.800 .500,00$ & $275.907,78$ & $4.208 .024,57$ \\
\hline Sudeste & $1.044 .227,99$ & $3.185 .668,13$ & $4.826 .195,04$ & $16.405 .864,50$ & $29.834 .143,82$ & $11.113 .325,69$ & $13.675 .613,47$ \\
\hline Vitória (Espírito Santo) & $1.000 .329,49$ & $1.918 .898,63$ & $1.924 .200,64$ & $1.568 .516,06$ & $1.586 .248,25$ & $1.994 .866,47$ & $1.691 .382,71$ \\
\hline Belo Horizonte (Minas & $43.898,5$ & $1.266 .769,5$ & $30.000,00$ & $6.318 .152,34$ & $4.124 .384,86$ & $561.119,88$ & $2.809 .806,68$ \\
\hline \multicolumn{8}{|l|}{ Gerais) } \\
\hline \multicolumn{8}{|l|}{ Janeiro) } \\
\hline São Paulo (São Paulo) & - & - & $2.426 .300,00$ & $8.519 .196,10$ & $24.123 .510,71$ & $8.557 .339,34$ & $9.124 .649,06$ \\
\hline Sul & $1.008 .587,24$ & 2.499.999,59 & $2.565 .624,25$ & $4.009 .522,01$ & $4.741 .404,59$ & $3.381 .940,90$ & $3.866 .545,59$ \\
\hline Curitiba (Paraná) & - & $3.445,48$ & $91.919,25$ & $2.104,19$ & $638.954,21$ & $270.180,20$ & $2.025 .011,06$ \\
\hline $\begin{array}{l}\text { Porto Alegre (Rio Grande } \\
\text { do Sul) }\end{array}$ & $310.542,52$ & $1.618 .535,24$ & $2.137 .827,44$ & $2.826 .595,37$ & $2.987 .366,68$ & $2.156 .692,15$ & $1.790 .590,57$ \\
\hline Florianópolis (Santa & $698.044,72$ & $878.018,87$ & $335.877,56$ & $1.180 .822,45$ & $1.115 .083,70$ & $955.068,55$ & $50.943,96$ \\
\hline \multicolumn{8}{|l|}{ (atarina) } \\
\hline Centro-oeste & $2.943 .459,78$ & $5.166 .557,14$ & $9.720 .905,87$ & $12.205 .855,82$ & $10.008 .565,39$ & $10.974 .988,42$ & $13.884 .851,79$ \\
\hline Distrito Federal & - & - & - & $3.641 .403,91$ & $3.260 .072,70$ & $1.890 .607,96$ & $3.956 .141,41$ \\
\hline Goiânia (Goiás) & $2.160 .459,78$ & $2.378 .405,28$ & $6.999 .083,91$ & $4.549 .855,05$ & $5.891 .961,32$ & $5.710 .331,57$ & $4.397 .886,95$ \\
\hline Cuiabá (Mato Grosso) & - & $1.969 .151,86$ & $990.514,46$ & $1.631 .250,81$ & $568.416,37$ & $1.976 .837,11$ & $2.430 .847,52$ \\
\hline Campo Grande (Mato & $783.000,00$ & $819.000,00$ & $1.731 .307,50$ & $2.383 .346,05$ & $288.115,00$ & $139.7211,78$ & $3.099 .975,91$ \\
\hline Grosso do Sul) & & & & & & & \\
\hline
\end{tabular}

Fonte: Fundo Nacional de Desenvolvimento para Educação (FNDE), 2019. 
Tabela 3

Evolução da utilização de recursos financeiros * (\%) na compra de alimentos da agricultura familiar para o Programa Nacional de Alimentação Escolar (PNAE), segundo macrorregiões geográficas, capitais de estado e Distrito Federal. Brasil, 2011-2017.

\begin{tabular}{|c|c|c|c|c|c|c|c|}
\hline & 2011 & 2012 & 2013 & 2014 & 2015 & 2016 & 2017 \\
\hline Total & 3,4 & 8,1 & 10,5 & 15,6 & 19,0 & 14,6 & 16,5 \\
\hline Norte & 11,6 & 25,8 & 37,3 & 40,9 & 50,6 & 46,7 & 53,1 \\
\hline Rio Branco (Acre) & 39,3 & 28,3 & 39,3 & 21,8 & 43,9 & 35,1 & 43,7 \\
\hline Macapá (Amapá) & - & 66,5 & 20,9 & 0,3 & 35,3 & 44,9 & 45,8 \\
\hline Manaus (Amazonas) & 5,9 & 29,0 & 41,2 & 44,1 & 51,1 & 53,6 & 58,8 \\
\hline Belém (Pará) & 26,9 & 15,7 & 45,7 & 51,5 & 34,1 & 40,3 & 39,6 \\
\hline Porto Velho (Rondônia) & - & - & 20,0 & 6,6 & 56,8 & 39,0 & 23,4 \\
\hline Boa Vista (Roraima) & - & 19,1 & 17,1 & 79,1 & 78,7 & 100,0 & 234,1 \\
\hline Palmas (Tocantins) & 23,3 & 46,9 & 49,3 & 68,1 & 65,4 & 31,0 & 49,6 \\
\hline Nordeste & 2,2 & 9,6 & 10,4 & 11,6 & 15,1 & 15,7 & 23,3 \\
\hline São Luís (Maranhão) & - & - & 31,2 & - & 18,5 & 27,2 & 23,6 \\
\hline Teresina (Piauí) & 19,6 & 36,3 & 29,6 & 46,4 & 36,3 & 46,4 & 49,6 \\
\hline Fortaleza (Ceará) & - & 4,4 & 0,1 & 5,7 & 1,1 & 9,0 & 12,0 \\
\hline Natal (Rio Grande do Norte) & - & - & 1,8 & 4,8 & 8,2 & 11,0 & 4,8 \\
\hline João Pessoa (Paraíba) & 1,1 & - & 12,1 & - & 3,6 & 10,5 & 36,8 \\
\hline Recife (Pernambuco) & - & - & - & - & - & - & 12,6 \\
\hline Maceió (Alagoas) & - & - & 0,1 & - & 6,7 & 100,0 & 4,7 \\
\hline Aracaju (Sergipe) & - & 12,5 & 11,4 & 6,7 & 5,6 & 84,1 & 312,4 \\
\hline Salvador (Bahia) & 2,0 & 24,7 & 3,6 & 24,6 & 40,7 & 1,6 & 29,7 \\
\hline Sudeste & 0,8 & 2,1 & 2,6 & 9,3 & 15,2 & 6,1 & 6,1 \\
\hline Vitória (Espírito Santo) & 25,2 & 40,0 & 36,1 & 34,9 & 29,5 & 32,6 & 33,0 \\
\hline Belo Horizonte (Minas Gerais) & 0,3 & 7,1 & 0,2 & 29,7 & 18,7 & 2,7 & 11,0 \\
\hline Rio de Janeiro (Rio de Janeiro) & - & - & 0,7 & - & - & - & 0,1 \\
\hline São Paulo (São Paulo) & - & - & 2,7 & 9,0 & 23,9 & 10,8 & 7,8 \\
\hline Sul & 5,6 & 11,4 & 8,8 & 14,7 & 15,7 & 9,5 & 11,2 \\
\hline Curitiba (Paraná) & - & - & 0,5 & - & 3,7 & 1,3 & 10,2 \\
\hline Porto Alegre (Rio Grande do Sul) & 5,6 & 26,0 & 27,7 & 34,9 & 33,9 & 22,5 & 18,1 \\
\hline Florianópolis (Santa Catarina) & 30,1 & 30,7 & 8,8 & 33,7 & 27,4 & 22,6 & 1,1 \\
\hline Centro-oeste & 5,5 & 9,0 & 16,2 & 23,7 & 15,5 & 14,5 & 19,2 \\
\hline Distrito Federal & - & - & - & 12,9 & 9,1 & 4,2 & 9,5 \\
\hline Goiânia (Goiás) & 26,6 & 26,5 & 70,6 & 46,1 & 50,4 & 41,1 & 35,1 \\
\hline Cuiabá (Mato Grosso) & - & 33,5 & 17,3 & 28,7 & 8,7 & 28,2 & 34,5 \\
\hline Campo Grande (Mato Grosso do Sul) & 11,2 & 10,1 & 18,0 & 31,0 & 2,7 & 13,7 & 28,1 \\
\hline
\end{tabular}

* (valor utilizado na compra de alimentos da agricultura familiar / valor total repassado pelo FNDE) x 100.

Fonte: Fundo Nacional de Desenvolvimento para Educação (FNDE), 2019.

\section{Discussão}

A obrigatoriedade de usar pelo menos 30\% do recurso repassado pelo FNDE para aquisição de alimentos da agricultura familiar, proposta pela Lei no 11.947/2009, tem o objetivo de diminuir o consumo dos alimentos processados e ultraprocessados na alimentação escolar e incentivar o consumo de alimentos in natura ou minimamente processados ${ }^{9}$, regionais e saudáveis, contribuindo com a diminuição da prevalência de obesidade e excesso de peso na população escolar 10.

Em estudo realizado na cidade de São Paulo, em 2013, constatou-se que frutas e hortaliças eram os principais itens presentes nas chamadas púbicas do estado, o que pode favorecer o aumento do consumo desses alimentos 11. 
A compra de alimentos da agricultura familiar, conforme a Resolução de no 38 12, de 16 de julho de 2009, pode ser realizada por dispensa de licitação, utilizando-se chamadas públicas amplamente divulgadas, contendo quantidades e especificação de alimentos.

A aquisição de alimentos da agricultura familiar proporciona a criação de um canal de comercialização para o agricultor familiar, incentivo à renda, inclusão produtiva, cooperativismo e associações no meio rural 13, além do fornecimento de alimentos in natura 12, saudáveis e mais baratos, haja vista a não participação de intermediários na venda dos alimentos 14 .

Apesar dos benefícios da aquisição de agricultura familiar, o estudo evidenciou que, no período de 2011 a 2017, apenas um terço das capitais cumpriu a meta estabelecida, o que demonstra a necessidade de incentivar a produção de agricultura familiar e fiscalizar melhor os recursos empregados.

A não adequação de algumas capitais pode ser resultado da burocratização da documentação necessária ao pequeno produtor, pequena cooperação dos setores de nutrição, dificuldade no fornecimento de diversidade de alimentos, ausência de equipamentos que facilitem a produção e distribuição bem como a dificuldade de logística na entrega dos alimentos 15 .

Em pesquisa realizada em 2011, mediante utilização de questionário eletrônico, foi investigada a compra de alimentos da agricultura familiar pelo PNAE em 3.817 municípios do Brasil. Verificou-se que $44,4 \%$ dos municípios aplicaram $30 \%$ ou mais recursos do FNDE. A Região Sul apresentou maior frequência de municípios cumprindo o estabelecido pela legislação (67\%), enquanto a Norte, a menor adequação $(26,4 \%) 16$.

O presente estudo evidenciou a Região Norte como a única que apresentou adequação durante todo o período avaliado, com apenas uma capital (Porto Velho) sem atingir a meta recomendada. Tal fato pode ter ocorrido em razão de a avaliação ter sido realizada apenas nas capitais.

Por terem sido utilizados dados secundários, os resultados apresentados são sujeitos a eventuais imprecisões do sistema de informações do FNDE. Entretanto, ele é o espaço oficial de acesso público para consulta sobre o tema. Além disso, o fato de os dados terem sido examinados por macrorregiões e capitais, ainda que tenha oferecido um panorama geral da evolução da aquisição de alimentos da agricultura familiar no país, não permitiu uma análise mais detalhada segundo município.

Concluiu-se que, no período de 2011 a 2017, apenas um terço das capitais avaliadas utilizou adequadamente a porcentagem de recursos financeiros para a compra de alimentos da agricultura familiar, embora tenha sido percebido aumento gradual na compra de tais alimentos, sobretudo nas capitais do Norte e Nordeste do Brasil. Fazem-se necessários maiores incentivos à aquisição desses alimentos, fiscalização dos recursos disponibilizados, bem como capacitação dos gestores municipais, agricultores familiares e todos os envolvidos no PNAE. Sugere-se a ampliação desta análise para todos os municípios brasileiros, incluindo outras variáveis como produtos adquiridos, número de chamadas públicas realizadas, número de agricultores familiares participantes e avaliação da contribuição da agricultura familiar para o desenvolvimento sustentável local e regional. 


\section{Colaboradores}

L. R. S. Araujo trabalhou na concepção, coleta de dados, interpretação dos dados, redação e revisão do manuscrito. A. N. M. Brito auxiliou no processo de revisão e redação do manuscrito. M. T. P. Rodrigues e M. D. M. Mascarenhas auxiliaram na análise, revisão e redação do manuscrito. R. S. R. Moreira-Araujo trabalhou na concepção, interpretação, redação e revisão crítica do manuscrito.

\section{Informações adicionais}

ORCID: Lana Raysa da Silva Araujo (0000-00031224-5788); Andrea Nunes Mendes de Brito (0000-0002-8270-9018); Malvina Thais Pacheco Rodrigues (0000-0001-5501-0669); Márcio Dênis Medeiros Mascarenhas (0000-0001-5064-2763); Regilda Saraiva dos Reis Moreira-Araujo (00000002-3669-2358).

\section{Agradecimentos}

À mestranda do Programa de Pós-graduação em Ciências e Saúde, Universidade Federal do Piauí: Ianne Fernandes da Silva.

\section{Referências}

1. Oliveira TRPR, Sousa HC, Silva AP. Agricultura familiar na alimentação escolar: estudo de caso em dois municípios de Minas Gerais. Nutrire Rev Soc Bras Aliment Nutr 2013; 38:25668.

2. Sousa AA, Silva APF, Azevedo E, Ramos MO. Cardápios e sustentabilidade: ensaio sobre as diretrizes do Programa Nacional de Alimentação Escolar. Rev Nutr PUCCAMP 2015; 28:217-29.

3. Pedraza DF, Melo NLSD, Silva FA, Araujo EMN. Avaliação do Programa Nacional de Alimentação Escolar: revisão da literatura. Ciênc Saúde Colet 2018; 23:1551-60.

4. Ministério da Educação; Fundo Nacional de Desenvolvimento da Educação; Coordenação Geral do Programa Nacional de Alimentação Escolar; Grupo Nacional de Direitos Humanos, Conselho Nacional dos Procuradores Gerais do Ministério Público dos Estados, do Distrito Federal e da União. Cartilha nacional de alimentação escolar. Brasília: Ministério da Educação/Fundo Nacional de Desenvolvimento da Educação/Coordenação Geral do Programa Nacional de Alimentação Escolar/ Grupo Nacional de Direitos Humanos, Conselho Nacional dos Procuradores Gerais do Ministério Público dos Estados, do Distrito Federal e da União; 2015.

5. Peixinho AML. A trajetória do Programa de Alimentação Escolar no período de 20032010: relato do gestor nacional. Ciênc Saúde Colet 2013; 18:909-16.

6. Cunha WAD, Freitas AFD, Salgado RJSF. Efeitos dos programas governamentais de aquisição de alimentos para a agricultura familiar em Espera Feliz, MG. Revista de Economia e Sociologia Rural 2017; 55:427-44.

7. Brasil. Lei no 11.947, de 16 de junho de 2009. Dispõe sobre o atendimento da alimentação escolar e do Programa Dinheiro Direto na Escola aos alunos da educação básica; altera as Leis no 10.880, de 9 de junho de 2004, 11.273, de 6 de fevereiro de 2006, 11.507, de 20 de julho de 2007; revoga dispositivos da Medida Provisória no 2.178-36, de 24 de agosto de 2001, e a Lei no 8.913, de 12 de julho de 1994; e dá outras providências. Diário Oficial da União 2009; 17 jun.

8. Fundo Nacional de Desenvolvimento da Educação. Resolução/CD/FNDE no 26, de 17 de junho de 2013. Dispõe sobre o atendimento da alimentação escolar aos alunos da educação básica no âmbito do Programa Nacional de Alimentação Escolar - PNAE. Diário Oficial da União 2013; 18 jun.

9. Triches RM, Kilian L. Papel dos atores sociais na aquisição de produtos da agricultura familiar para alimentação escolar em municípios paranaenses. Redes 2016; 21:159-79.

10. Triches RM, Schneider S. Alimentação escolar e agricultura familiar: reconectando o consumo à produção. Saúde Soc 2010; 19:933-45. 
11. Amorim ALB, Rosso VV, Bandoni DH. Acquisition of family farm foods for school meals: analysis of public procurements within rural family farming published by the cities of São Paulo state. Rev Nutr PUCCAMP 2016; 29:297-306.

12. Fundo Nacional de Desenvolvimento da Educação. Resolução/CD/FNDE no 38, de 16 de julho de 2009. Dispõe sobre o atendimento da alimentação escolar aos alunos da educação básica no Programa Nacional de Alimentação Escolar (PNAE). Diário Oficial da União 2009; 17 jun.

13. Fundo Nacional de Desenvolvimento da Educação. Manual de aquisição de produtos da agricultura familiar para a alimentação escolar. 2a Ed. Brasília: Fundo Nacional de Desenvolvimento da Educação; 2016.
14. Hawkes C, Brazil BG, Castro IRR, Jaime PC. How to engage across sectors: lessons from agriculture and nutrition in the Brazilian School Feeding Program. Rev Saúde Pública 2016; 50:47.

15. Toyoyoshi JY, Oliveira R, Santos MSN, Galisa MS, Galante AP. Avaliação da aquisição de gêneros alimentícios provenientes da agricultura familiar para a alimentação escolar. Mundo Saúde 2013; 37:329-35.

16. Machado PMO, Schmitz BAS, Gonzáles-Chica DA, Corso ACT, Vasconcelos FAG, Gabriel CG. Compra de alimentos da agricultura familiar pelo Programa Nacional de Alimentação Escolar (PNAE): estudo transversal com o universo de municípios brasileiros. Ciênc Saúde Colet 2018; 23:4153-64. 


\section{Abstract}

The study aimed to verify the adequacy of funds for food purchases from family farming in the scope of the National School Food Program (PNAE) in Brazil's state capitals and the Federal District. This was a descriptive study based on secondary data obtained from the webpage of the National Fund for the Development of Education (FNDE) in the link dedicated to information on family farming. We analyzed the amounts transferred to the FNDE and the percentage used in purchasing foods from family farmers for school meals in the state capitals and Federal District from 2011 to 2017. Data were collected in May 2019. One-third of the 27 cities (33.3\%) reported purchases above the minimum recommended level (30\%). The North of Brazil complied with the recommended level (39.4\%), while the Southeast reported the lowest level (6.4\%). There was an increase from 2011 to 2017 in the total amounts invested and in the number of cities that purchased foods from family farming. Boa Vista (Roraima) was the capital that spent the largest share of its school meal funds on foods produced by family farmers (56.6\%). In 2017, the local governments in Boa Vista and Aracaju (Sergipe) offered matching funds for the funds transferred from the FNDE for these food purchases. In conclusion, from 2011 to 2017, only one-third of the capital cities adequately used the percentage of funds for purchasing foods from family farming, although there was a gradual increase in the purchase of these foods, especially in the state capitals from North and Northeast Brazil.

Agriculture; School Feeding; Government Financing

\section{Resumen}

El objetivo de este estudio fue verificar la adecuación de la utilización de recursos financieros para la compra de alimentos procedentes de la agricultura familiar, dentro del ámbito del Programa $\mathrm{Na-}$ cional de Alimentación Escolar (PNAE), en capitales de estados brasileños y en el Distrito Federal. Se trata de un estudio descriptivo con análisis de datos secundarios, obtenidos de la página electrónica del Fondo Nacional de Desarrollo para la Educación (FNDE), dentro de la sección dedicada a la información sobre agricultura familiar. Se investigaron los valores proporcionados por el FNDE y el porcentaje destinado a la compra de alimentos de agricultura familiar para la adquisición en escuelas de capitales de estados brasileños, así como en el Distrito Federal, durante el período de 2011 a 2017. Los datos se recogieron en mayo de 2019. De las 27 ciudades evaluadas, cerca de un tercio $(33,3 \%)$ presentó una compra por encima del mínimo recomendado (30\%). La Región Norte cumplió lo recomendado (39,4\%), mientras que la Sudeste presentó el menor porcentaje de compra (6,4\%). De 2011 a 2017, se percibió un aumento en los valores totales invertidos y en el número de ciudades compradoras de alimentos procedentes de agricultura familiar. Entre las capitales evaluadas, Boa Vista (Roraima) fue la que más utilizó recursos para la adquisición de alimentos de agricultura familiar (56,6\%). En 2017, los ayuntamientos de Boa Vista y Aracaju (Sergipe) ofrecieron una partida financiera adicional, además de los recursos proporcionados por el FNDE, para la compra de estos alimentos. Se concluye que, durante el período de 2011 a 2017, solamente un tercio de las capitales evaluadas utilizó adecuadamente el porcentaje de recursos financieros para la compra de alimentos de la agricultura familiar, aunque se haya percibido un aumento gradual en la compra de estos alimentos, especialmente en las capitales del Norte y Nordeste de Brasil.

Agricultura; Alimentación Escolar; Financiación Gubernamental
Recebido em 09/Jan/2019

Versão final reapresentada em 01/Ago/2019

Aprovado em 14/Ago/2019 